\title{
Pirfenidone treatment of idiopathic pulmonary fibrosis
}

This article was published in the following Dove Press journal:

Therapeutics and Clinical Risk Management

8 February 20II

Number of times this article has been viewed

Ye Gan ${ }^{1,2}$

Erica L Herzog ${ }^{2}$

Richard H Gomer ${ }^{3}$

'Department of Medicine, Central South University, Changsha, Hunan, China; ${ }^{2}$ Department of Medicine, Yale University School of Medicine, New Haven, CT, USA; ' ${ }^{2}$ epartment of Biology, Texas A\&M University, College Station, TX, USA
Correspondence: Richard H Gomer Department of Biology, Texas A\&M University, ILSB MS 3474, College Station, TX 77843-3474, USA

Tel + I 9794585745

Fax + | $979845289 \mid$

Email rgomer@tamu.edu

\begin{abstract}
Idiopathic pulmonary fibrosis (IPF) is a discrete clinicopathologic entity defined by the presence of usual interstitial pneumonia on high-resolution CT scan and/or open lung biopsy and the absence of an alternate diagnosis or exposure explaining these findings. There are currently no FDA-approved therapies available to treat this disease, and the 5-year mortality is $\sim 80 \%$. The pyridone derivative pirfenidone has been studied extensively as a possible therapeutic agent for use in this deadly disease. This review will present the unique clinical features and management issues encountered by physicians caring for IPF patients, including the poor response to conventional therapy. The biochemistry and preclinical efficacy of pirfenidone will be discussed along with a comprehensive review of the clinical efficacy, safety, and side effects and patient-centered foci such as quality of life and tolerability. It is hoped that this information will lend insight into the complex issues surrounding the use of pirfenidone in IPF and lead to further investigation of this agent as a possible therapy in this devastating disease.
\end{abstract}

Keywords: pirfenidone, fibrosis, clinical trials

\section{Introduction to management issues for idiopathic pulmonary fibrosis}

In fibrosing diseases, regions of tissue that resemble scar tissue form in inappropriate locations such as the heart, lungs, and liver. There are at least 62 different fibrosing diseases, and collectively these are associated with $\sim 45 \%$ of deaths in the United States. ${ }^{1}$ The lung manifests multiple forms of fibrosis. Many of these diseases are associated with a secondary diagnosis such as underlying autoimmune disease or gastrointestinal reflux. ${ }^{2}$ Occupational exposures, such as asbestos and heavy metals, are another important cause of lung fibrosis. 'Idiopathic pulmonary fibrosis' (IPF) is defined as fibrosis in the setting of a high-resolution CT scan pattern and/or lung biopsy consistent with usual interstitial pneumonia (UIP) and lack of a known risk factor for interstitial lung disease. ${ }^{2}$ IPF affects $\sim 130,000$ patients in the United States and has a 5 -year mortality rate of $80 \%{ }^{3}$ This review will focus on the management aspects of this discrete clinicopathologic diagnosis and the potential use of pirfenidone in this patient population.

\section{Importance of accurate diagnosis}

Establishing an accurate diagnosis for the patient who presents with interstitial lung disease is an essential component of management. ${ }^{2}$ The initial workup of pulmonary fibrosis includes a complete history and physical examination. A detailed occupational history should be elicited along with detailed questions regarding gastrointestinal reflux 
and rheumatologic symptoms such as myopathic changes or Raynaud's phenomenon. While recent American Thoracic Society (ATS) guidelines recommend against routine use of bronchoscopy in the diagnosis of IPF, serologic evaluation to uncover occult autoimmune disease remains an important aspect of the initial evaluation. ${ }^{2}$ High-resolution CT scan remains the cornerstone of diagnosis, with the pathognomic changes of UIP being peripheral, basilar predominance of fibrosis, honeycombing, and an absence of other findings such as a ground-glass appearance or nodules that might suggest an alternate diagnosis. ${ }^{2}$ Should the patient presentation be inconsistent with UIP/IPF, an alternate diagnosis may be sought with the use of open lung biopsy. Biopsy readings require the temporal heterogeneity of established fibrosis, fibroblastic foci, and normal-appearing lung parenchyma to be considered consistent with UIP. ${ }^{2}$ Findings of bronchocentricity, granulomas, and/or predominant inflammation are generally considered 'inconsistent' with UIP, and in this case, the patient should be diagnosed as 'not UIP/IPF'. While revised diagnostic criteria were presented by Raghu et al at the 2010 meeting of the ATS, these recommendations have not yet been published and are not presented here. The decision to pursue biopsy should be considered in light of the potential for clinical decline following this invasive procedure since some studies suggest that open lung biopsy can lead to accelerated respiratory failure and even death. ${ }^{4}$ An especially helpful aspect of patient diagnosis is the use of multidisciplinary teams for the initial diagnosis of IPF. These teams include trained pulmonologists, pathologists, and radiologists and, in some cases, a rheumatologist. ${ }^{5}$

\section{Pulmonary function testing}

Once the diagnosis of UIP/IPF has been established, a number of patient care issues require ongoing attention. Patients are generally followed with serial measurements of forced vital capacity (FVC) and diffusion capacity of carbon monoxide (DLCO). Of these, the percent predicted FVC is most reflective of the degree of ventilatory impairment as the DLCO can reflect vascular changes in addition to parenchymal pathology. ${ }^{6}$ Six-minute walk tests (6MWTs) are also used but reflect overall patient conditioning rather than restrictive physiology. In addition, the clinical utility of data obtained from this measure remains uncertain. ${ }^{7}$ Total lung capacity (TLC) is used less frequently because of the relatively preserved chest wall recoil and inspiratory muscle function. ${ }^{8}$ Reductions in FVC and DLCO are associated with poor survival, 9,10 and longitudinal decline in these parameters portends an even worse prognosis. ${ }^{11}$
Oxygen saturation $\left(\mathrm{SpO}_{2}\right)$ is another important parameter of disease severity and is used to guide the decision to institute supplemental oxygen therapy. ${ }^{12}$

\section{Management of gastrointestinal reflux disease}

Effective management of the patient with IPF includes attention to conditions that are commonly associated with this disease. For example, gastrointestinal reflux is frequently found in IPF patients and may be a contributing factor to this disease. ${ }^{13,14}$ Intriguingly, a preliminary study with four IPF patients indicated that treatment of reflux can lead to stabilization of the FVC decline. ${ }^{15}$ Workup of GERD may include a barium swallow, endoscopy, and $\mathrm{pH}$ probe. Management may range from simple lifestyle modifications to pharmacologic therapy with histamine receptor blockers or proton pump inhibitors. In some extreme cases, a Nissen fundoplication may be warranted. ${ }^{16}$ Consultation with an esophageal specialist may be indicated for particularly challenging cases.

\section{Acute exacerbations}

IPF demonstrates significant associations with other cardiopulmonary disorders including coronary artery disease, ${ }^{17,18}$ pulmonary embolism, ${ }^{19}$ sleep apnea, ${ }^{20}$ and lung cancer. ${ }^{21}$ In addition, patients with IPF suffer heightened manifestations of respiratory infections. Thus, these comorbidities should be considered when evaluating the IPF patient who manifests clinical worsening. However, it is now recognized that patients with IPF are at risk for 'acute exacerbations', a clinical syndrome defined as a new ( $<1$ month) increase in oxygen requirement, new ground-glass findings on CT, and diffuse alveolar damage pattern on histopathology. ${ }^{22,23}$ These exacerbations account for up to $50 \%$ of deaths in IPF and may affect previously stable patients. ${ }^{24}$ Management of acute exacerbations includes ruling out possible infection (bacterial and viral) and, if clinically indicated, evaluation for life-threatening conditions such as coronary artery disease and pulmonary embolism. ${ }^{22}$ There exists no consensus regarding medical therapies in acute exacerbations. One small retrospective study reported that high-dose corticosteroids followed by cyclosporine may be beneficial in this population, ${ }^{25}$ although this treatment approach has not been studied systematically. Another small study reported that long-term use of Coumadin prevented acute exacerbations in patients with $\mathrm{IPF},{ }^{26}$ which may relate to modulation of the recently reported profibrotic effects of coagulation factors, although the generalizability of these results (which were 
primarily obtained from inpatients) to outpatients seen in the clinic remains uncertain. Given the high mortality associated with acute exacerbations, they are considered legitimate targets for prevention and therapy. A possible role for pirfenidone in reducing the incidence of acute exacerbations will be discussed in the following sections.

\section{Lung transplantation}

One particularly important aspect of management is early referral for lung transplantation. Because IPF may progress rapidly, early referral to a transplant center is a crucial aspect of management. Lung transplantation is the only therapy shown to increase survival in IPF. ${ }^{27}$ While it has been suggested that the rate of patient decline should trigger early referral for orthotopic lung transplantation, ${ }^{28}$ the inability to predict which patients will develop an accelerated worsening and respiratory failure mandates that transplant referral be considered early on in the clinical course regardless of apparent stability. ${ }^{29}$ Compared with single lung transplantation, bilateral lung transplantation may confer improved outcomes such as bronchiolitis obliterans syndrome-free survival and increased 1-year survival. ${ }^{30}$ In addition, single lung transplantation in patients with pulmonary hypertension may increase the risk of primary graft dysfunction. ${ }^{31}$

\section{Pharmacologic therapy}

The results of pharmacologic trials for IPF have been disappointing. Interferon gamma, which had in subgroup analysis of prior studies shown possible benefit on reducing the FVC decline in patients with early stage disease ${ }^{32}$ and overall mortality, ${ }^{33}$ failed to demonstrate similar benefits in a multicenter, placebo-controlled trial. ${ }^{34}$ Similarly, the endothelin antagonist bosentan demonstrated a trend toward improved symptoms and delayed time to death in a randomized trial of IPF patients, ${ }^{35}$ but this benefit was not seen in a recently completed larger phase III trial. ${ }^{36} \mathrm{High}$-dose $N$-acetylcysteine showed a modest but significant effect on preservation of FVC when used in combination therapy with prednisone and azathioprine compared to those patients randomized to prednisone and azathioprine only ${ }^{37}$ However, because prednisone and azathioprine are not routinely used in the treatment of IPF, the benefit of $N$-acetylcysteine as monotherapy is unknown. The ongoing National Institutes of Health (NIH)-sponsored PANTHER trial is examining this question. Etanercept, a recombinant receptor for tumor necrosis factor $\alpha$ (TNF- $\alpha$ ) with neutralizing activity, was tested in a 48-week-long randomized controlled trial of 88 IPF patients that compared $25 \mathrm{mg}$ etanercept twice weekly with placebo. While there was no difference in the primary outcome measure of percent-predicted FVC, DLCO, or resting $\mathrm{PaO}_{2}$, post-hoc analysis revealed a trend toward reduced disease progression in the etanercept-treated cohort. ${ }^{38}$ This study (which many thought was well designed but underpowered) ( $^{39}$ may lead to larger trials examining the role of etanercept in IPF. ${ }^{36}$ The recent NIH-sponsored Sildenafil Trial of Exercise Performance in Idiopathic Pulmonary Fibrosis, which examined the effect of sildenafil on the six-minute walking test in IPF patients with advanced disease, concluded that there was no physiologic improvement in patients receiving sildenafil. ${ }^{40}$ However, symptoms were greatly improved, which the authors felt would be of benefit to patients suffering from IPF. When viewed in combination, these results underscore the need for the development of novel therapies that are efficacious for use in IPF. For this reason, pirfenidone, which has shown promising results in preclinical studies, has been proposed for use in this difficult-to-treat population.

\section{History of pirfenidone}

Starting with the previous observation that some pyridones had analgesic properties, Gadekar investigated several pyridone derivatives and found that 5-methyl-1-phenyl-2(1H)-pyridone (which was then later named pirfenidone) had analgesic, antipyretic, and antiinflammatory activities and lowered serum uric acid and serum glucose with low toxicity. ${ }^{41}$ Later, apparently starting with the hypothesis that some anti-inflammatory drugs might be useful for the treatment of fibrosis, Margolin found that pirfenidone could function as an orally available antifibrotic in rats. ${ }^{42}$ Oral pirfenidone was also found to be effective as an antifibrotic in the bleomycininduced hamster pulmonary fibrosis model. ${ }^{43}$

InterMune, Inc purchased the patent rights for pirfenidone for the United States and Europe from Marnac, Inc in 2007. In Japan, pirfenidone is sold by Shionogi as Pirespa ${ }^{\circledR}$. In October 2010, the Indian drug company Cipla began sales of generic pirfenidone (trade name Pirfenex) in India for the treatment of IPF.

\section{Pirfenidone mode of action}

Despite impressive advances, much remains to be understood about the mechanism of action of pirfenidone. Pirfenidone inhibits cell proliferation, apparently by inhibiting DNA synthesis, in human myometrial and leiomyoma cells and also decreases levels of mRNAs encoding collagen I and collagen III in these cells. ${ }^{44}$ In human retinal pigment epithelial cells, pirfenidone inhibits a transforming growth factor (TGF)- $\beta 1$-induced increase in 
fibronectin synthesis. ${ }^{45}$ In a patent application, Margolin found that pirfenidone inhibited, among other things, fibroblast growth factor-induced fibroblast proliferation and TGF- $\beta 1$-induced collagen production from fibroblasts, ${ }^{46}$ and in a hamster model, pirfenidone decreased collagen gene expression. ${ }^{47}$ Pirfenidone reduces prolyl hydroxylase activity in the lungs of hamsters treated with bleomycin, but has no effect on prolyl hydroxylase activity in vitro. ${ }^{47}$ However, the structure of pirfenidone (Figure 1) resembles some prolyl hydroxylase inhibitors. ${ }^{48}$ Pirfenidone decreases levels of intercellular adhesion molecule-1 on fibroblasts. ${ }^{49}$ In a hamster model of pulmonary fibrosis, pirfenidone decreased levels of platelet-derived growth factor. ${ }^{50}$ In a murine macrophage-like cell line, pirfenidone suppressed translation of the proinflammatory cytokine TNF- $\alpha,{ }^{51}$ and in mice with lipopolysaccharide (LPS)-induced endotoxic shock, oral pirfenidone decreased plasma levels of the proinflammatory cytokines interferon- $\gamma$ and interleukin- 6 and increased plasma levels of the antiinflammatory cytokine interleukin-10. ${ }^{51}$ In a murine bleomycin-induced pulmonary fibrosis model, pirfenidone increased the lung content of interferon- $\gamma$ and decreased levels of monocyte chemoattractant protein-1, stromal cell-derived factor- $1 \alpha$, TGF- $\beta 1$, basic fibroblast growth factor, interleukin-1 $\beta$, interleukin-6, interleukin-12p40, and interleukin-18. ${ }^{52}$ In cell culture, pirfenidone inhibits T-cell activation and proliferation. ${ }^{53}$ In vitro, pirfenidone scavenges hydroxyl radicals. ${ }^{54}$ Taken together, the plethora of effects of pirfenidone point to its promise as having multiple beneficial effects on fibrosis. However, this multitude of effects also makes it difficult to determine its exact mechanism of action. Given the promise of pirfenidone, a considerable amount of work remains to be done to elucidate how it works, and this work should uncover additional targets that may be useful to develop next-generation antifibrotics.

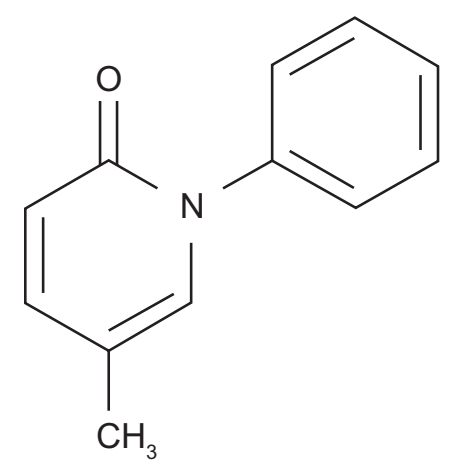

Figure I The structure of pirfenidone. After ingestion, the $\mathrm{CH}_{3}$ group at the bottom of the structure is rapidly metabolized to primarily $\mathrm{COOH}$ and to a lesser extent $\mathrm{OH}$.

\section{Pirfenidone pharmacology and pharmacokinetics}

In healthy human volunteers, orally administered pirfenidone is absorbed within 20-60 min and cleared from the serum in 2-2.5 h, with food intake somewhat decreasing the absorption.$^{55}$ However, coadministration with food appears to improve tolerability in older adults. ${ }^{56}$ In humans, pirfenidone is rapidly metabolized to primarily 5 -carboxy pirfenidone (where the methyl group at the bottom of Figure 1 is oxidized to a $\mathrm{COOH}$ group and to a lesser extent 5-hydroxy pirfenidone, where the methyl group is replaced with an $\mathrm{OH}) .{ }^{56}$ These metabolites are then excreted in the urine. Since pirfenidone is rapidly metabolized, determining the effect of the metabolites on specific enzymatic activities, such as prolyl hydroxylase activity, needs to be determined.

\section{Pirfenidone is effective in other disease models}

Pirfenidone attenuated sclerosing peritonitis in a rat model ${ }^{57}$ and was also effective in rat models of renal failure, ${ }^{58,59}$ hepatic fibrosis, ${ }^{60}$ and cardiac fibrosis. ${ }^{61}$ When human keloid tissues were implanted in nude mice, oral pirfenidone decreased the implant weights. ${ }^{62}$ Pirfenidone inhibited LPS-induced toxic shock in a mouse model ${ }^{63}$ and was partially effective in decreasing ischemia/reperfusion-induced injury in a rat small intestine model. ${ }^{64}$ Finally, topical application of a $10 \%$ pirfenidone solution three times a day for 7 days after thermoplasty-induced foreleg lameness in horses decreased swelling and heat and increased flexion. ${ }^{65}$ Together, these observations suggest that pirfenidone might be a useful therapeutic for several different diseases.

\section{Efficacy studies}

The initial human data supporting a role for pirfenidone in the treatment of IPF was published by Raghu et al in 1999. ${ }^{66} \mathrm{In}$ this phase II study, 54 patients with severe IPF, many of whom were actively taking immunosuppressive medications such as prednisone, Imuran, or Cytoxan, were recruited from the University of Washington Interstitial Lung Disease Program and treated with open-label pirfenidone at a divided dose of $3600 \mathrm{mg} /$ day over a 25 -month period. During this time, the 1 -year mortality was $22 \%$ and the 2 -year mortality was $37 \%$. The authors compared these results to other published studies of IPF outcomes and found the comparison to be favorable. In terms of physiologic derangements, the authors found that over the study period, the trends for FVC and DLCO (for those patients able to perform them) remained stable. As the study progressed, fewer patients were able to complete 
pulmonary function tests (PFTs) (at entry, all 54 patients performed this analysis, 6 months into the study, only 41 patients were able to perform PFTs, and by 12 months, only 31 patients performed PFTs). Thus, this stability in PFTs fails to reflect the clinical worsening experienced by a substantial number of patients, which the authors themselves pointed out. Another endpoint, cessation of immunosuppressive therapy, found that the majority of patients were able to discontinue prednisone or azathioprine. Oxygen requirements appeared to remain stable after 12 months of therapy, and there was one patient who actually improved enough to completely discontinue supplemental oxygen. Side effects were relatively minor and will be discussed in the following sections. While in retrospect, this study had many shortcomings, including the small sample size recruited from only one medical center, the concomitant use of immunosuppression (which by today's standards is not standard of care), and the lack of a control group; the results were encouraging enough to warrant further human investigation in both the United States and abroad.

In a study published in 2005, Azuma and colleagues examined the efficacy of divided dose $1800 \mathrm{mg} /$ day pirfenidone in 107 Japanese patients with IPF who were recruited to take part in this randomized, placebo-controlled phase II trial. ${ }^{67}$ This study randomized patients to pirfenidone or placebo at a 2:1 drug:placebo ratio. The primary endpoint was change in baseline on mean percent $\mathrm{SpO}_{2}$ on a six-minute exercise test (6MET) that was personalized for each patient's speed and comfort. Secondary endpoints included PFTs, HCRT patterns, frequency of acute exacerbations, and serum levels of KL-6, a marker of pneumocyte damage. Interestingly and somewhat unexpectedly, 25 patients were unable to perform the 6MET upon trial entry. Thus, the investigators opted to use this measurement only in the patients who could perform it on study entry.

The results for the primary endpoint of this study were negative in that for all comers, there was no difference in the lowest $\mathrm{SpO}_{2}$ of the $6 \mathrm{MET}$ between those patients receiving placebo or control. However, subgroup analysis revealed that of the patients who could complete the 6MET, pirfenidone demonstrated a significant increase in mean $\mathrm{SpO}_{2}$. Regarding secondary endpoints, the 6-month change in vital capacity (VC) between placebo and pirfenidone treated was negligible but by 9 months, the difference was greater and reached statistical significance $(-0.13 \mathrm{~L}$ vs $-0.01 \mathrm{~L}, P<0.036)$. Changes in TLC, diffusion capacity, $\mathrm{PaO}_{2}$, HRCT severity, dyspnea, quality of life, and serum KL-6 were not affected by the administration of pirfenidone. However, despite this seeming lack of benefit, interim data analysis performed at the 9-month time point revealed a statistically significant reduction in acute exacerbations in the pirfenidone-treated group compared to control (14\% vs $0 \%, P<0.0003)$. This apparent benefit prompted the data safety monitoring board to discontinue the study early.

While some authors questioned the choice of endpoints and the decision to end the trial early, ${ }^{68,69}$ the results of this second phase II trial were encouraging enough to prompt several phase III trials. Taniguchi et al have recently published the results of a multicenter, phase III placebo-controlled trial performed in Japan. ${ }^{70}$ In this study, 267 patients with IPF were randomized to receive 52 weeks of pirfenidone at high dose (1800 mg/day), low dose (1200 mg/day), or placebo. The primary endpoint, which was changed before unblinding, was change in $\mathrm{VC}$ between weeks 0 and 52. Secondary outcomes were progression-free survival (with progression defined as death or $<10 \%$ decline in VC) or inability to perform VC due to acute exacerbation or symptoms. Tertiary endpoints included PFTs, acute exacerbation, biomarker measurements of alveolar damage such as surfactant-associated protein A, surfactant-associated protein D and KL-6 levels (a mucin-like glycoprotein), and symptoms. Demographics were largely similar between groups, although a nearly significant increase in smokers was detected in the $1800 \mathrm{mg}$ /day group $(P=0.067)$.

Importantly, the primary endpoint was attained in that patients treated with $1800 \mathrm{mg}$ /day of pirfenidone (when compared to placebo) demonstrated a significant attenuation of $\mathrm{VC}$ decline measured as both absolute values $(-0.09 \mathrm{~L}$ vs $-0.16 \mathrm{~L}, P=0.042)$ and percent predicted $(-2.91$ vs $-5.13 \%, P=0.044)$. Progression-free survival was also improved in the $1800 \mathrm{mg}$ treated group $(P=0.028)$. Encouragingly, when compared to the placebo subjects, the $1200 \mathrm{mg} /$ day group also displayed reduced $\mathrm{VC}$ decline $(-0.08 \mathrm{~L}$ vs $-0.16 \mathrm{~L}, P=0.039)$. Neither the $1800 \mathrm{mg} /$ day nor the $1200 \mathrm{mg}$ /day recipients demonstrated improvements in the other measured endpoints. Based on these encouraging results (which were not without some methodologic flaws ${ }^{71,72}$ ), pirfenidone was approved for monotherapy treatment of IPF in Japan.

The results of two American Phase III trials were presented at the 2009 meeting of the ATS. ${ }^{36}$ The first of these, PIPF-004, was a phase III randomized, placebo-controlled, 72-week trial that had three arms: high-dose pirfenidone (2403 mg/day), low-dose pirfenidone (1197 mg/day), and placebo. Enrollment proceeded in a 2:1:2 ratio and included a primary outcome of absolute change in percent-predicted FVC between enrollment and week 72 . Secondary endpoints 
included progression-free survival, worsened IPF, categorical change in percent predicted FVC, symptom measurements, percent-predicted DLCO, six-minute walk distance, and nadir $\mathrm{SpO}_{2}$. The exploratory endpoints assessed subjects for changes in quality of life, new oxygen requirement, all-cause mortality, and number of days without a hospitalization for respiratory reasons. When the data were unblinded, it was found that baseline characteristics of patients were similar throughout all three arms and that most patients (78\% per group) finished the study. Importantly, a $157 \mathrm{~mL}(3.06 \%)$ reduction in percent-predicted FVC decline over the 72-week study period was noted in the patients receiving high-dose pirfenidone compared to control $(P=0.001)$. The high-dose group also exhibited improvement in two secondary endpoints: fewer patients experiencing a categorical decline in percent-predicted FVC and more patients demonstrating improved progression-free survival. The results of the low-dose arm were reported to demonstrate a 'dose response effect', but the primary data are not available at this time.

In order to expand these promising results, InterMune undertook another 72-week phase III trial (PIPF006) comparing the effect of high-dose pirfenidone to placebo. This study randomized 344 patients in a 1:1 ratio and again used the primary endpoint of absolute change in percent-predicted FVC between weeks 0 and 72. The measured secondary endpoints assessed progression-free survival, worsened IPF, categorical change on percent-predicted FVC, dypsnea measurements, percent-predicted DLCO, distance achieved during 6MWT, and nadir $\mathrm{SpO}_{2}$. Exploratory outcomes were all-cause mortality, new oxygen requirement, number of days without a hospitalization for respiratory causes, and objective measures of quality of life.

Patients in this study were matched in terms of clinically relevant demographics and indices of severity. Approximately $80 \%$ of patients completed treatment in both groups, with death or adverse events being the most common cause for dropout. ${ }^{36}$ Unfortunately, a beneficial effect primary endpoint of percent decline in percent-predicted FVC was not achieved $(P=0.501)$, although there was a significant treatment effect at weeks 12, 24, 36, and 48 of the study ( $P<0.05$ at these time points). Further analysis revealed that the effect of high-dose pirfenidone on percentpredicted FVC decline at 72 weeks was similar to that seen in PIPF004, but that there was a more robust placebo effect in the PIPF006 study. The only secondary endpoint to be significant was the distance attained in the 6MWT.

These findings were interpreted by the sponsor, InterMune, as being clinically significant and prompted an application to the Food and Drug Administration (FDA) for approval of pirfenidone for the treatment of IPF. The application was actually granted fast track approval, and the advisory panel recommended approval. Closer examination of the panel's recommendation revealed that while 9/12 panel members recommended approval of pirfenidone, the efficacy vote was $7 / 12$, indicating that fewer panel members thought that the drug was efficacious. Thus, it may not have surprised many observers when the FDA rejected the application in early May 2010. One concern was the perceived lack of efficacy and the lack of survival benefit (http://www.fda. gov/downloads/AdvisoryCommittees/CommitteesMeet ing Materials/Drugs/Pulmonary-AllergyDrugsAdvisory Committee/UCM208806.pdf). It is currently not clear whether future trials will be conducted.

\section{Safety and tolerability}

The safety profile of pirfenidone is excellent, and it appears to be generally well tolerated. The 1999 study by Raghu et $\mathrm{al}^{66}$ reported a high rate of adverse complaints related to pirfenidone $(87 \%)$ though most of these were relatively minor as only $11 \%$ of subjects actually discontinued the drug. The most common effects were gastrointestinal upset (64\%), fatigue $(42 \%)$, and a plethora of dermatologic problems including photosensitivity (24\%). ${ }^{66}$ In the 2005 Azuma study, which included a placebo arm, $98.5 \%$ of patients receiving pirfenidone reported any type of adverse symptoms compared to $88.9 \%$ in the placebo arm $(P<0.04) .{ }^{67}$ These included photosensitivity ( $43.8 \%$ vs $0 \%, P<0.000)$, gastrointestinal upset (30.1\% vs $8.3 \%, P<0.01)$, nausea $(21.9 \%$ vs $5.6 \%$, $P<0.0314)$, anorexia (31.5\% vs 5.6\%, $P<0.0030)$, and fatigue $(21.9 \%$ vs $2.8 \%, P<0.0102)$. Despite this high rate of adverse events, only $15.1 \%$ of patients receiving pirfenidone discontinued the study drug versus $5.6 \%$ in the placebo arm. This difference did not meet statistical significance $(P=0.2132) .{ }^{67}$ In the 2009 Taniguchi study, which utilized both high- and low-dose pirfenidone compared to placebo, nearly all subjects experienced some adverse events (100\% vs $98.1 \%$ vs $99.1 \%, P$ values NS). Photosensitivity was significantly more common in the pirfenidone-treated subjects regardless of dose (high dose $51.4 \%$ vs low dose $52.7 \%$ vs placebo $22.4 \%$ ). Whereas anorexia was more pronounced in the high-dose group (16.5\% vs $10.9 \%$ vs $2.8 \%$ ), abdominal discomfort was more prevalent in the low-dose subjects $(7.3 \%$ vs $2.8 \%$ high dose vs $0.0 \%$ ). Dizziness and liver enzyme abnormalities were also seen with increased frequency in the high-dose group. Similar to earlier studies, these adverse events did not appear to prompt discontinuation of the drug 
as withdrawal rates were similar in all three arms (18.3\% vs $20 \%$ vs $13.1 \%)^{70}$ The preliminary results of PIPF004 and PIPF006 both report increased adverse event-related study withdrawal in the high-dose group $;{ }^{36}$ however, because these results have not been published, the full data are not available for review at this time.

\section{Patient-focused perspectives such as quality of life, patient satisfaction/acceptability, adherence, and uptake}

There exist only limited data that evaluate pirfenidone's effects on patient satisfaction and quality of life. While the 1999 Raghu study did not examine quality of life as an endpoint, the four other studies (Azuma, Taniguchi, PIPF004, and PIPF006) did include symptoms and objective measures of quality of life. In all of these studies, the reported results do not demonstrate an improvement in quality of life measures. However, these data also indicate that pirfenidone does not negatively impact quality of life in patients with early or late stage IPF. In addition, the increase in side effects appears to have been tolerable for many patients, as despite the high rate of adverse events, the frequency of drug discontinuation was actually quite low. Thus, from these data, one can infer that pirfenidone is reasonably well tolerated.

\section{Summary}

Pirfenidone is an intriguing new drug for the treatment of IPF, with approval in Japan and further sales in India. A wide variety of effects on cells has made understanding the mode of action difficult, and further studies on the basic cell biology and biochemistry of this drug will greatly expand our understanding of pulmonary fibrosis. Emerging data suggest that pirfenidone may be beneficial in mitigating $\mathrm{FVC}$ decline in patients with mild-to-moderate disease, and the side effects profile appears tolerable. It is thus hoped by many that future studies will demonstrate that pirfenidone represents a new era in the treatment of a currently fatal disease.

\section{Acknowledgments}

We thank Dr Tadhg Begley for helpful suggestions and Lisa Cooper for drawing Figure 1. This work was funded in part by NIH HL083029 and funds from the Scleroderma Research Foundation and the American Thoracic Society.

\section{Disclosure}

YG and ELH have no disclosures relevant to this report. RHG is a science advisory board member for Trellis Bioscience and has received royalties and stock options from Trellis. Trellis is developing new antibody therapeutics and is not involved with pulmonary fibrosis or pirfenidone. He is also a cofounder and one of the science advisory board members for Promedior and has received royalties and stock options from Promedior. Promedior is developing serum amyloid $\mathrm{P}$ as a therapeutic for fibrosing diseases, including idiopathic pulmonary fibrosis, and is not involved in any work with or marketing of pirfenidone.

\section{References}

1. Wynn TA. Fibrotic disease and the $\mathrm{T}(\mathrm{H}) 1 / \mathrm{T}(\mathrm{H}) 2$ paradigm. Nat Rev Immunol. 2004;4(8):583-594.

2. American Thoracic Society; European Respiratory Society. American Thoracic Society/European Respiratory Society International Multidisciplinary Consensus Classification of the Idiopathic Interstitial Pneumonias. This joint statement of the American Thoracic Society (ATS), and the European Respiratory Society (ERS) was adopted by the ATS board of directors, 2001 Jun and by the ERS Executive Committee, 2001 Jun. Am J Respir Crit Care Med. 2002;165(2):277-304.

3. Raghu G, Weycker D, Edelsberg J, Bradford WZ, Oster G. Incidence and prevalence of idiopathic pulmonary fibrosis. Am J Respir Crit Care Med. 2006;174(7):810-816.

4. Yuksel M, Ozyurtkan MO, Bostanci K, Ahiskali R, Kodalli N. Acute exacerbation of interstitial fibrosis after pulmonary resection. Ann Thorac Surg. 2006;82(1):336-338.

5. Castelino FV, Goldberg H, Dellaripa PF. The impact of rheumatological evaluation in the management of patients with interstitial lung disease. Rheumatology (Oxford). 2010 Aug 4. Epub ahead of print.

6. Martinez FJ, Flaherty K. Pulmonary function testing in idiopathic interstitial pneumonias. Proc Am Thorac Soc. 2006;3(4): 315-321.

7. Swigris JJ, Wamboldt FS, Behr J, et al. The 6 minute walk in idiopathic pulmonary fibrosis: longitudinal changes and minimum important difference. Thorax. 2010;65(2):173-177.

8. Nava S, Rubini F. Lung and chest wall mechanics in ventilated patients with end stage idiopathic pulmonary fibrosis. Thorax. 1999;54(5): 390-395.

9. Hubbard R, Johnston I, Britton J. Survival in patients with cryptogenic fibrosing alveolitis: a population-based cohort study. Chest. 1998; 113(2):396-400.

10. Jezek V, Fucik J, Michaljanic A, Jezkova L. The prognostic significance of functional tests in cryptogenic fibrosing alveolitis. Bull Eur Physiopathol Respir. 1980;16(6):711-720.

11. Flaherty KR, Andrei AC, Murray S, et al. Idiopathic pulmonary fibrosis: prognostic value of changes in physiology and six-minute-walk test. Am J Respir Crit Care Med. 2006;174(7):803-809.

12. Ryu JH, Daniels CE. Advances in the management of idiopathic pulmonary fibrosis. F1000 Med Rep. 2010;2:28.

13. Tobin RW, Pope CE 2nd, Pellegrini CA, Emond MJ, Sillery J, Raghu G. Increased prevalence of gastroesophageal reflux in patients with idiopathic pulmonary fibrosis. Am J Respir Crit Care Med. 1998;158(6): 1804-1808.

14. Raghu G, Freudenberger TD, Yang S, et al. High prevalence of abnormal acid gastro-oesophageal reflux in idiopathic pulmonary fibrosis. Eur Respir J. 2006;27(1):136-142.

15. Raghu G, Yang ST, Spada C, Hayes J, Pellegrini CA. Sole treatment of acid gastroesophageal reflux in idiopathic pulmonary fibrosis: a case series. Chest. 2006;129(3):794-800.

16. Gaude GS. Pulmonary manifestations of gastroesophageal reflux disease. Ann Thorac Med. 2009;4(3):115-123.

17. Nathan SD, Basavaraj A, Reichner C, et al. Prevalence and impact of coronary artery disease in idiopathic pulmonary fibrosis. Respir Med. 2010;104(7):1035-1041. 
18. Hubbard RB, Smith C, Le Jeune I, Gribbin J, Fogarty AW. The association between idiopathic pulmonary fibrosis and vascular disease: a populationbased study. Am J Respir Crit Care Med. 2008;178(12):1257-1261.

19. Panos RJ, Mortenson RL, Niccoli SA, King TE Jr. Clinical deterioration in patients with idiopathic pulmonary fibrosis: causes and assessment. Am J Med. 1990;88(4):396-404.

20. Lancaster LH, Mason WR, Parnell JA, et al. Obstructive sleep apnea is common in idiopathic pulmonary fibrosis. Chest. 2009;136(3): $772-778$.

21. Hubbard R, Venn A, Lewis S, Britton J. Lung cancer and cryptogenic fibrosing alveolitis. A population-based cohort study. Am J Respir Crit Care Med. 2000;161(1):5-8.

22. Collard HR, Moore BB, Flaherty KR, et al. Acute exacerbations of idiopathic pulmonary fibrosis. Am J Respir Crit Care Med. 2007;176(7): 636-643.

23. Hyzy R, Huang S, Myers J, Flaherty K, Martinez F. Acute exacerbation of idiopathic pulmonary fibrosis. Chest. 2007;132(5):1652-1658.

24. Martinez FJ, Safrin S, Weycker D, et al; IPF Study Group. The clinical course of patients with idiopathic pulmonary fibrosis. Ann Intern Med. 2005;142(12 Pt 1):963-967.

25. Homma S, Sakamoto S, Kawabata M, et al. Cyclosporin treatment in steroid-resistant and acutely exacerbated interstitial pneumonia. Intern Med. 2005;44(11):1144-1150.

26. Kubo H, Nakayama K, Yanai M, et al. Anticoagulant therapy for idiopathic pulmonary fibrosis. Chest. 2005;128(3):1475-1482.

27. Algar FJ, Espinosa D, Moreno P, et al. Results of lung transplantation in idiopathic pulmonary fibrosis patients. Transplant Proc. 2010;42(8): 3211-3213.

28. Mackay LS, Anderson RL, Parry G, Lordan J, Corris PA, Fisher AJ. Pulmonary fibrosis: rate of disease progression as a trigger for referral for lung transplantation. Thorax. 2007;62(12):1069-1073.

29. Wuyts WA, Thomeer M, Dupont LJ, Verleden GM. An algorithm for referral of patients with IPF for lung transplantation. Thorax. 2008; 63(3):292; author reply 292.

30. Neurohr C, Huppmann P, Thum D, et al; Munich Lung Transplant Group. Potential functional and survival benefit of double over single lung transplantation for selected patients with idiopathic pulmonary fibrosis. Transpl Int. 2010;23(9):887-896.

31. Fang A, Studer S, Kawut SM, et al; for the Lung Transplant Outcomes Group. Elevated pulmonary artery pressure is a risk factor for primary graft dysfunction following lung transplantation for idiopathic pumonary fibrosis. Chest. 2010 Sep 23. Epub ahead of print.

32. Raghu G, Brown KK, Bradford WZ, et al; Idiopathic Pulmonary Fibrosis Study Group. A placebo-controlled trial of interferon gamma-1b in patients with idiopathic pulmonary fibrosis. N Engl J Med. 2004; 350(2):125-133.

33. Ziesche R, Hofbauer E, Wittmann K, Petkov V, Block LH. A preliminary study of long-term treatment with interferon gamma-1b and low-dose prednisolone in patients with idiopathic pulmonary fibrosis. $N$ Engl J Med. 1999;341(17):1264-1269.

34. King TE Jr, Albera C, Bradford WZ, et al; INSPIRE Study Group. Effect of interferon gamma-1b on survival in patients with idiopathic pulmonary fibrosis (INSPIRE): a multicentre, randomised, placebocontrolled trial. Lancet. 2009;374(9685):222-228.

35. King TE Jr, Behr J, Brown KK, et al. BUILD-1: a randomized placebocontrolled trial of bosentan in idiopathic pulmonary fibrosis. Am J Respir Crit Care Med. 2008;177(1):75-81.

36. Behr J, Noble PW. Clinical trials in interstitial lung disease. European Respiratory Monograph. 2009;46:67-84.

37. Demedts M, Behr J, Buhl R, et al; IFIGENIA Study Group. High-dose acetylcysteine in idiopathic pulmonary fibrosis. $N$ Engl $J$ Med. 2005;353(21):2229-2242.

38. Raghu G, Brown KK, Costabel U, et al. Treatment of idiopathic pulmonary fibrosis with etanercept: an exploratory, placebo-controlled trial. Am J Respir Crit Care Med. 2008;178(9):948-955.

39. Jackson RM, Fell CD. Etanercept for idiopathic pulmonary fibrosis: lessons on clinical trial design. Am J Respir Crit Care Med. 2008;178(9):889-891.
40. Zisman DA, Schwarz M, Anstrom KJ, Collard HR, Flaherty KR, Hunninghake GW; Idiopathic Pulmonary Fibrosis Clinical Research Network. A controlled trial of sildenafil in advanced idiopathic pulmonary fibrosis. N Engl J Med. 2010;363(7):620-628.

41. Gadekar SM, inventor. 5-Methyl-1-phenyl-2-(1H)-pyridone compositions and methods of use. US patent 3,974,281. 1976 Aug 10.

42. Margolin SB, inventor. Composition and method for reparation and prevention of fibrotic lesions. US patent 5,310,562. 1994 May 10.

43. Iyer SN, Wild JS, Schiedt MJ, Hyde DM, Margolin SB, Giri SN. Dietary intake of pirfenidone ameliorates bleomycin-induced lung fibrosis in hamsters. J Lab Clin Med. 1995;125(6):779-785.

44. Lee BS, Margolin SB, Nowak RA. Pirfenidone: a novel pharmacological agent that inhibits leiomyoma cell proliferation and collagen production. J Clin Endocrinol Metab. 1998;83(1):219-223.

45. Zhang S, Shiels IA, Ambler JS, Taylor SM. Pirfenidone reduces fibronectin synthesis by cultured human retinal pigment epithelial cells. Aust N Z J Ophthalmol. 1998;26 Suppl 1:S74-S76.

46. Margolin SB, inventor. Treatment of cytokine growth factor caused disorders. US patent 6,090,822. $2000 \mathrm{Jul} 18$.

47. Iyer SN, Gurujeyalakshmi G, Giri SN. Effects of pirfenidone on procollagen gene expression at the transcriptional level in bleomycin hamster model of lung fibrosis. J Pharmacol Exp Ther. 1999;289(1): 211-218.

48. Nangaku M, Izuhara Y, Takizawa S, et al. A novel class of prolyl hydroxylase inhibitors induces angiogenesis and exerts organ protection against ischemia. Arterioscler Thromb Vasc Biol. 2007;27(12): $2548-2554$.

49. Kaneko M, Inoue H, Nakazawa R, et al. Pirfenidone induces intercellular adhesion molecule-1 (ICAM-1) down-regulation on cultured human synovial fibroblasts. Clin Exp Immunol. 1998;113(1):72-76.

50. Gurujeyalakshmi G, Hollinger MA, Giri SN. Pirfenidone inhibits PDGF isoforms in bleomycin hamster model of lung fibrosis at the translational level. Am J Physiol. 1999;276(2 Pt 1):L311-L318.

51. Nakazato H, Oku H, Yamane S, Tsuruta Y, Suzuki R. A novel antifibrotic agent pirfenidone suppresses tumor necrosis factor-alpha at the translational level. Eur J Pharmacol. 2002;446(1-3):177-185.

52. Oku H, Shimizu T, Kawabata T, et al. Antifibrotic action of pirfenidone and prednisolone: different effects on pulmonary cytokines and growth factors in bleomycin-induced murine pulmonary fibrosis. Eur J Pharmacol. 2008;590(1-3):400-408.

53. Visner GA, Liu F, Bizargity P, et al. Pirfenidone inhibits T-cell activation, proliferation, cytokine and chemokine production, and host alloresponses. Transplantation. 2009;88(3):330-338.

54. Misra HP, Rabideau C. Pirfenidone inhibits NADPH-dependent microsomal lipid peroxidation and scavenges hydroxyl radicals. $\mathrm{Mol}$ Cell Biochem. 2000;204(1-2):119-126.

55. Shi S, Wu J, Chen H, Wu J, Zeng F. Single- and multiple-dose pharmacokinetics of pirfenidone, an antifibrotic agent, in healthy Chinese volunteers. J Clin Pharmacol. 2007;47(10):1268-1276.

56. Rubino CM, Bhavnani SM, Ambrose PG, Forrest A, Loutit JS. Effect of food and antacids on the pharmacokinetics of pirfenidone in older healthy adults. Pulm Pharmacol Ther. 2009;22(4):279-285.

57. Suga H, Teraoka S, Ota K, et al. Preventive effect of pirfenidone against experimental sclerosing peritonitis in rats. Exp Toxicol Pathol. 1995;47(4):287-291.

58. Shimizu T, Fukagawa M, Kuroda T, et al. Pirfenidone prevents collagen accumulation in the remnant kidney in rats with partial nephrectomy. Kidney Int Suppl. 1997;63:S239-S243.

59. Shimizu T, Kuroda T, Hata S, Fukagawa M, Margolin SB, Kurokawa K. Pirfenidone improves renal function and fibrosis in the post-obstructed kidney. Kidney Int. 1998;54(1):99-109.

60. Tada S, Nakamuta M, Enjoji M, et al. Pirfenidone inhibits dimethylnitrosamine-induced hepatic fibrosis in rats. Clin Exp Pharmacol Physiol. 2001;28(7):522-527.

61. Nguyen DT, Ding C, Wilson E, Marcus GM, Olgin JE. Pirfenidone mitigates left ventricular fibrosis and dysfunction after myocardial infarction and reduces arrhythmias. Heart Rhythm. 2010;7(10):1438-1445. 
62. Shetlar MR, Shetlar DJ, Bloom RF, Shetlar CL, Margolin SB. Involution of keloid implants in athymic mice treated with pirfenidone or with triamcinolone. J Lab Clin Med. 1998;132(6):491-496.

63. Cain WC, Stuart RW, Lefkowitz DL, Starnes JD, Margolin S, Lefkowitz SS. Inhibition of tumor necrosis factor and subsequent endotoxin shock by pirfenidone. Int J Immunopharmacol. 1998;20(12):685-695.

64. Arumugam TV, Shiels IA, Margolin SB, Taylor SM, Brown L. Pirfenidone attenuates ischaemia-reperfusion injury in the rat small intestine. Clin Exp Pharmacol Physiol. 2002;29(11):996-1000.

65. Giri SN, Margolin SB. Effects of topical application of pirfenidone ointment on thermoplasty-induced acute lameness in a double-blind and acute and chronic lameness of musculoskeletal origin in an open multi-centered field trial in horses. Res Commun Mol Pathol Pharmacol. 2005;117-118:47-63.

66. Raghu G, Johnson WC, Lockhart D, Mageto Y. Treatment of idiopathic pulmonary fibrosis with a new antifibrotic agent, pirfenidone: results of a prospective, open-label Phase II study. Am J Respir Crit Care Med. 1999;159(4 Pt 1):1061-1069.
67. Azuma A, Nukiwa T, Tsuboi E, et al. Double-blind, placebo-controlled trial of pirfenidone in patients with idiopathic pulmonary fibrosis. Am J Respir Crit Care Med. 2005;171(9):1040-1047.

68. Mathai SC, Polito AJ. The questionable efficacy of pirfenidone in IPF. Am J Respir Crit Care Med. 2005;172(9):1228.

69. Levitt J, Gould MK. Poor choice of primary outcome in a clinical trial of pirfenidone in patients with IPF. Am J Respir Crit Care Med. 2005;172(9):1228-1229; author reply 1229.

70. Taniguchi H, Ebina M, Kondoh Y, et al; Pirfenidone Clinical Study Group in Japan. Pirfenidone in idiopathic pulmonary fibrosis. Eur Respir J. 2010;35(4):821-829.

71. Collard HR. Idiopathic pulmonary fibrosis and pirfenidone. Eur Respir J. 2010;35(4):728-729.

72. Swigris J, Fairclough D. Pirfenidone in idiopathic pulmonary fibrosis. Eur Respir J. 2010;36(3):695-696; author reply 696-698.
Therapeutics and Clinical Risk Management

\section{Publish your work in this journal}

Therapeutics and Clinical Risk Management is an international, peerreviewed journal of clinical therapeutics and risk management, focusing on concise rapid reporting of clinical studies in all therapeutic areas, outcomes, safety, and programs for the effective, safe, and sustained use of medicines. This journal is indexed on PubMed Central, CAS,

\section{Dovepress}

EMBase, Scopus and the Elsevier Bibliographic databases. The manuscript management system is completely online and includes a very quick and fair peer-review system, which is all easy to use. Visit http://www.dovepress.com/testimonials.php to read real quotes from published authors.

Submit your manuscript here: http://www.dovepress.com/therapeutics-and-clinical-risk-management-journal 